\title{
Muenstraia, ein neues Rugosa-Genus (Anthozoa) aus dem Obersilur und Unterdevon
}

\author{
Dieter Weyer ${ }^{1}$ \\ Mit 3 Abbildungen und 1 Tafel
}

\section{Zusammenfassung}

Muenstraia n. gen. ist eine der ältesten ahermatypischen Rugosa (Subordo Cyathaxoniina) und umfasst neben der Typusart Muenstraia franconica n. sp. (Ludlovium, Elbersreuther Orthoceratitenkalk, Frankenwald) drei weitere Arten: Muenstraia squarrosa (Sutherland, 1965) (unteres Ludlovium, Henryhouse-Formation, Oklahoma), Muenstraia sp. (oberes Lochkovium. Yukon-Gebiet), Muenstraia thuringica n. sp. (Pragium, Thüringisches Schiefergebirge und Tafilalt). Die Gattung kann von dem isolierten, nur aus Xinjiang bekannten Protozaphrentis Yü, 1957 des hohen Mittelordoviz abgeleitet werden: wichtige Deszendenten im Ludlovian sind Laccophyllum Simpson, 1900 und Sutherlandinia Weyer, 1972.

Der Bauplan entspricht dem seit Schindewolf (1931) traditionellen Konzept der Gattung Petraia Münster, 1839, die aber nach Revision (Weyer 2000) ihrer wahren Typusart Petraia decussata Münster, 1839 aus dem oberen Famennium einer anderen Entwicklungsrcihe angehört (Neaxoninae Hill, 1981, jetzt Petraiidae Koninck, 1872). Für die dadurch namenlos gewordene Familia „Petraiidae“ (etwa sensu Hill 1981) werden die bisher als Synonym ruhenden Protozaphrentidae Ivanovskiy, 1959 verfügbar, denen noch Duncanella Nicholson, 1874 sowie die Sutherlandiniinae Weyer, 1972 und die Ditoecholasmatinae Sutherland. 1965 zugeordnet sind.

Schlüsselwörter: ahermatypische Korallen, Cyathaxoniina-Taxonomie, Frankenwald und Thüringisches Schiefergebirge (Saxothuringikum, Deutschland), Tafilalt (Marokko).

\author{
Summary \\ Muenstraia, a new genus of Rugosa (Anthozoa) from the Late Silurian and Early Devonian
}

The new taxon, one of the most ancient members of the ahermatypic suborder Cyathaxoniina, includes the type species Muenstraia franconica n. sp. (Ludlovian, Elbersreuth Orthoceratites-Limestone Formation, Upper Franconia, Germany) and three further species: Muenstraia squarrosa (Sutherland, 1965) (lower Ludlovian, Henryhouse Formation, Oklahoma. USA), Muenstraia sp. (upper Lochkovian, Yukon Territories, Canada), Muenstraia thuringica n. sp. (middle/upper Pragian, Tentaculitid Limestonc Formation, Thuringian Mountains, Germany, and middle Pragian, Tafilalt, Morocco). The genus descends from the isolated Upper Middle Ordovician Protozaphrentis Yü, 1957, only known from Xinjiang in China; it is the ancestor of two new phylogenetic lines starting in the Ludlovian with Laccophyllum Simpson, 1900, and Sutherlandinia Weyer, 1972.

Morphology and diagnosis are identical with the (since Schindewolf 1931) traditional definition of the genus Petraia Münster, 1839, which represents according to a revision of its real and Upper Famennian type species Petraia decussata Münster. 1839 (Weycr 2000) another phylogenetic line (Neaxoninae Hill, 1981, now Petraiidae Koninck, 1872). Therefore, the valid name of the family "Petraiidae" (sensu Hill 1981) becomes Protozaphrentidae Ivanovskiy, 1959, which comprise also Duncanella Nicholson, 1874, and both the Sutherlandiniinae Weyer, 1972 and Ditoecholasmatinae Sutherland, 1965.

Key words: ahermatypic corals, taxonomy of Cyathaxoniina, Upper Franconia and Thuringian Mountains (Germany), Tafilalt (Morocco).

\section{Einleitung}

Die Stammesgeschichte der ältesten ahermatypischen Rugosa (Subordo Cyathaxoniina) ist nur lückenhaft bekannt; die bisherigen, im Detail stark voneinander abweichenden Klassifikationen können nur momentane Näherungsversuche sein.
Hindernisse für einen besseren Kenntnisstand sind die Seltenheit entsprechender Faunen aus psychrosphärischer Fazies im Ordoviz und Silur, die wenigen verfügbaren Studien dieser kleinwüchsigen Formenkreise und vielfach fehlende Revisionen älterer Taxa mit moderner Serienschlifftechnik. Besonders frappierend wirkt das

Löwestrasse 15, D-10249 Berlin, Germany. E-Mail: dieter.weyer@t-online.de Erhaiten März 2001, angenommen Juni 2001. 


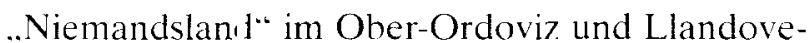
rium (zwischer den Unikaten Protozaphrentis Yü. 1957 des hohen Mittel-Ordoviz und Duncanella Nicholson. 18:4 im Wenlockium): erst im OberSilur (Ludlovium) ..erscheinen“ zusätzliche Genera, erste kleine Assoziationen (Laccophyllum. Columnaxon. Neaxon. ..Petraia". Sutherlandinia. Ditoecholasma. Palaeocyathus, Oligophyllum. Anisophyllum - beispielsweise Henryhouse-Formation. Oklah sma: Sutherland 1965).

Das älteste Familia-Taxon (sensu Hill 1981: F187). die Petraiidae Koninck. 1872. hat seine morphologisch-taxonomische Basis verloren. nachdem Petrita Münster. 1839 erneut zu revidieren war (Wever 2000): 79 - Ober-FamenniumDeszendent v.on Neaxon Kullmann. 1965). Die bisherige Grundlage (Petraia-Revision von Schindewolf 1931) ərwies sich als völlig verfehlt und unbrauchbar: fehlende Fixierung aller PetraiaSyntypen durch Designation von intensiv mit Serienschliffen untersuchten Lectotypen (speziell für die wahre Typusart Petraia decussata Münster, 1839), ur richtige Ausgangshypothese. dass im Elbersreuther Orthoceratiten-Kalk nur ein Rugosa-Genus mit lediglich zwei Arten vorkäme (unerkannt blieb die vorrangige Präsenz von $P a-$ laeocyathus Foerste. 1888. dem der Lectotypus von Petraia ra tiata Münster. 1839 angehört). unzulässige Dek arationen abweichender Polypare als "abnormal individual", die real andere Gattungen reprisentieren (Schindewolf 1931: Abb. $17-18==$ Laccophyllum. Lectotypus von Petraia semistriata Münster. 1839. Taf. 52. Fig. 6 = unbekannte; Genus).

Der traditionelle morphologische Petraia-Maßstab war das on Schindewolf (1931: Abb. 5-9) mitgeteilte Polypar aus deutschem Ludlovium unglücklicherweise kein Syntypus aus der Kollektion Münster (1839). So urteilten auch Hill (1956: F259) and Weyer (1978: 100, 1980: 30). Dagegen wäh te Hill (1981: F187) ein anderes Polypar von :ichindewolf (1931: Abb. 1-4) als Bezugsbasis, das wegen seines diploseptalen Antiseptums als Sutherlandinia sp. gedeutet wurde (Weyer 1972: 453, 1978: 93. 1980: 31). Dieser ..Petraia"-Bauplan ist zurzeit generisch heimatlos und wird hier als neue Gattung Muenstraia vorgestellt. von der weiterhin eine neue Art aus deutschem und marokkanischem Pragium vorliegt sowie zwisi Vertreter aus dem Unter-Ludlovium und $\mathrm{Ob} \curvearrowright$ r-Lochkovium Nordamerikas beschrieben sinc. Eine solche regional und auch stratigraphisch disjunkte Verbreitung deutet schon an, dass zusätzliche Vorkommen des noch seltenen Taxor s weltweit zu erwarten sind.
Folgende Abkürzungen werden verwendet: MB Berlin, Museum für Naturkunde der Humboldt-Universität zu Berlin: BGR Berlin. Bundesanstalt für Geologie und Rohstoffe, Außenstelle Berlin (früher: Preußische Geologische Landesanstalt. Zentrales Geologisches Institut): n, Anzahl der Großsepten: N. Anzahl aller Septen: D, Durchmesser. In den Querschliffzeichnungen, die sämtlich seitenrichtig zueinander orientiert sind, wurden 6 Protosepten markiert.

\section{Subordo Cyathaxoniina Spasskiy, 1977}

Die veränderte Nomenklatur (sensu Weyer 1996: 85) verwendet diesen Namen (statt Metriophyllina Spasskiy. 1965 sensu Hill, 1981) für die Unterordnung. weil Metriophyllum Milne-Edwards \& Haime. 1850 (nach einer Revision von Rohart \& Wever, in Vorbereitung) zu den „Stereolasmatina Hill, 1981" (Familia Lindstroemiidae Počta, 1902) = Zaphrentoidina Schouppé \& Stacul, 1959 gehört.

In Unkenntnis von Spasskiy (1977) wurde noch einmal eine angeblich neue Ordo Cyathaxoniida errichtet (Lin \& Liu 1999), die merkwürdigerweise neben den Cyathaxoniidae und Ekvasophyllidae auch die Amygdalophyllidae einschließen soll, obwohl bereits ein SubordoName Amygdalophyllina Wang, Ouyang \& Yan [in Lin et al.]. 1995 existiert.

Die Autorschaft könnte man auch Volz (1896: 105 - ..Stamm" Cyathaxonoidea) zuschreiben, denn es gibt vergleichbare Fälle (Hill 1981), wo Taxa der Ordo-Gruppe akzeptiert werden, obwohl sie primär nicht als Ordo-Niveau deklariert waren (..sectio" Cystiphyllida Nicholson, 1989). Andererseits ist bei konfuser Hierarchie auch schon ein Name verworfen worden (Hill 1981: F261 - Subordo Columnariina Soshkina, 1941 statt ..sub-order Columnariae" Rominger, 1876). Außerdem schließt der „Internationale Code der Zoologischen Nomenklatur" Namen oberhalb der Familia-Gruppe leider immer noch nicht in sein strenges Regelwerk ein.

\section{Familia Protozaphrentidae Ivanovskiy, 1959}

Dieser bisher als Synonym der Petraiidae Koninck. 1872 (sensu Hill 1981) ruhende FamiliaName ist zu reaktivieren, nachdem sich Petraia Münster. 1839 als direkter Deszendent von Neaxon Kullmann, 1965 und Glied der Neaxoninae Hill. 1981 erwies, die daraufhin unerwartet zum jüngeren Synonym der Petraiidae werden.

Ivanovskiy (1959) hatte die Familie für die Nominatgattung und zwei neue Genera (Tungussophyllum. Archaeozaphrentis) errichtet; sie sollte 
von den Streptelasmatidae Nicholson, 1889 abstammen und homöomorphe Ähnlichkeit zu den Hapsiphyllidae Grabau, 1928 des Unterkarbon zeigen. Später hielt Ivanovskiy (1963: 49) Protozaphrentis für enger mit den Streptelasmatidae als mit den Zaphrentoididae verwandt, erklärte Archaeozaphrentis zum nomen dubium und verwies Tungussophyllum in eine eigene neue, den Hapsiphyllidae nahe stehende Familia Tungussophyllidae. Tungussophyllum Ivanovskiy, 1959 ist nach Weyer (1974) ein Synonym von Rhegmaphyllum Wedekind, 1927 und gehört zu den Densiphyllidae Dybowski, 1873 (in der Nähe der Streptelasmatidae Nicholson, 1889). Bei Ivanovskiy (1965: 59) ist Protozaphrentis dann auch als Genus der Streptelasmatidae aufgeführt und die Familie Protozaphrentidae damit wieder eingezogen.

Alle diese Urteile stützten sich auf die unbrauchbare Originaldiagnose von Yü (1957), die inzwischen radikal umgedeutet wurde (Weyer 1973: Umkehr der Protosepten-Orientierung, Einordnung in die "Petraiidae" alter Fassung).

Die Subfamilia-Gliederung folgt annähernd Weyer (1978) und lautet:

Protozaphrentinae Ivanovskiy, 1959

Protozaphrentis Yü, 1957, Duncanella Nicholson, 1874. Muenstraia nov. gen.

Sutherlandiniinae Weyer, 1972 (mit Diplosepten)

Sutherlandinia Wcycr, 1972, Haptophyllum Pedder, 1967. Boolelasma Pedder, 1967, Pedderelasma Weyer, 1972. Petraiella Rózkowska, 1969

Ditoecholasmatinae Sutherland, 1965 (mit Diplosepten und Axialsynapticulae)

Ditoecholasma Simpson, 1900

Dass dieses System teilweise subjektiv und vorerst nicht konsequent phylogenetisch ist (Fedorowski 1984), dürfte selbstverständlich sein (speziell angesichts des oft noch mangelhaften Kenntnisstandes). Auf mögliche Polyphylie und Einzelfälle typisch „horizontaler“ Systematik bei den Sutherlandiniinae hatte ich selbst schon hingewiesen (Weyer 1978: 93). Vom Standard des Rugosa-Septalapparats abweichende Baupläne wie die Diplosepten (Weyer 1997a) der Sutherlandiniinae und Ditoecholasmatinae oder die axial bifurcaten Großsepten der sicherlich auch heterogenen "Subordo“ Diffingiina Fedorowski, 1986 sind so merkwürdig, dass zunächst die Monophylie in diesem Merkmal plausibel erscheint; oft stellt sich später heraus, dass tatsächlich polyphyletische Trends in unabhängigen, meist aber nahe verwandten Entwicklungsreihen vorliegen.

Bezeichnend für die Protozaphrentidae und besonders für ihre ältesten Vertreter (Protozaphrentis, Muenstraia) ist die eigenartige Mischung plesiomorpher und apomorpher Merkmale. Erstere sind der tiefe Kelch, die direkte axiale Septenverbindung, das lange Cardinalseptum ohne Fossula; zu den Letzteren zählen die contratingenten Kleinsepten und das biforme Tabularium. Die andere große, im Kleinsepten-Bau primitivere Cyathaxoniida-Familie, die Petraiidae Koninck, 1872 (Synonym Neaxoninae Hill, 1981) ist scheinbar erst ab unterem Ludlovium bekannt [Neaxon adaensis (Sutherland, 1965)]. muss aber parallel zu Protozaphrentis offensichtlich auch schon im Ordoviz existiert haben.

Als mit den Protozaphrentidae am engsten verwandte, von ihnen abstammende Familie werden die Cyathaxoniidac Milne-Edwards \& Haime, 1850 angesehen, die sich wie folgt gliedern lassen:

Laccophyllinae Grabau, 1928

Laccophyllum Simpson. 1900. Bitraia Galle \& Weyer, 1973, Thuriantha Weyer, 1981

Taralasmatinae Hill, 1981

Schindewolfia Weissermel, 1943, Retiophvllum Počta. 1902, Taralasma Pedder, 1967

Columnaxoninae Weyer, 1980

Columnaxon Scrutton, 1971

Cyathaxoniinae Milne-Edwards \& Haime, 1850

Cyathaxonia Michelin, 1847, Cyathocarinia Soshkina, 1928

Wesentlich erscheint hier die Vereinigung von Laccophyllinae und Cyathaxoniinae in einer einzigen Familia, denn Cyathaxonia ist ein im unteren Famennium entstandener direkter Deszendent von Laccophyllum (mit dem einzigen Unterschied des zur Columella herausgehobenen everten Aulos). Dieser aseptale Columella-Typ tritt völlig isoliert nur hier auf, während sonstige karbonisch-permische, leider immer mit den Cyathaxoniidae in Verbindung gebrachte Rugosa-Taxa stets primär septale Axialstrukturen besitzen, an deren Aufbau vorrangig das Antiseptum beteiligt ist.

\section{Muenstraia n. gen.}

Diagnose: Kleine konische Polypare mit tiefem Kelch und sehr dünnen Septen. Großsepten axial direkt verbunden, radial gestellt; Kleinsep- 
ten relativ larg und contratingent (mit betonter antiseptaler Triade). Cardinalseptum an konvexer Polyparse te. lang. auch im mittleren Kelch unverkürzt. Fseudofossulae mitunter und vorübergehend (nach Septeninsertionen) deutlich. oft aber nicht erkennbar. Septenmikrostruktur vermutlich lamellär (Septalränder glatt. ohne Dornen). Tab slarium kräftig biform. in Position II zur Peripherie hin abfallend und nur nahe der Polyparspitze entwickelt. in Position I flach (zentripetal t:icht abfallend oder auch ansteigend), hoch ir den Kelch hinaufreichend.

Derivatio nominis: nach Gcorg Graf zu Münster (1776-1844) und in Anlehnung an das von ihm 1839 aufgestellte, meist fehlinterpretierte (Schintlewolf 1931) und erst kürzlich (Weyer 2000 redefinicrte Rugosa-Genus Petraia.

\section{Ty pusart: Mluenstraia franconica $\mathrm{n}$. $\mathrm{sp}$.}

Beziehungt:n: Beim gegenwärtigen Kenntnisstand lässt sic 1 die neue Gattung nur von Protozaphrentis Yü. 1957 aus dem hohen Mittelordoviz ableiten: Unterschiede sind die dort dicken Septen mit suinösen Flanken und trabekulärer Mikrostruktur und dort fehlende Tabulae.

Als unmitt $\epsilon$ lbare Deszendenten im Ludlovium kommen zwei Genera in Betracht. zunächst Laccophyllum Sirupson, 1900 mit wohl entwickeltem beständigen Aulos, sowie Sutherlandinia Weyer. 1972 mit Diplosepten (Anti- und Cardinalseptum. antiseptale Kleinsepten). Das letztere Genus besitzt in der Regel einen markanten Aulos. aber bei eingen leider noch ungenügend bekannten. kün tig vielleicht einmal generisch abzutrennenden Arten kommt offensichtlich auch noch eine dir skte Großseptenverbindung im Polyparzentrum wie bei Muenstraia vor (speziell Sutherlandinic sp. Weyer 1980): 31. Abb. 3/1-3 $=$..Petraia semistriata". Schindewolf 1931: Abb. 1-4. als Begleiter von Muenstraia franconica n. sp. an deren locus typicus).

Bemerkun!son: Das neue Taxon entspricht dem traditionellen Perraia-Konzept (seit Schindewolf 1931). Lieser Bauplan besaß keinen gültigen Gattung:namen mehr. nachdem die echte Petraia Müns er. 1839) redefiniert wurde (Weyer 2000: Wahl und Beschreibung eines Lectotypus der Typusart Petraia decussata Münster. 1839 aus dem Ober-Fámennium vom Fundort Schübelhammer im Frankenwald).

Die angebliche trabekuläre Septenmikrostruktur von ..Petrita", die Weyer (1978: 94) vermutete, basiert auf Schindewolf's Abbildung (1931:
Taf. 52. Fig. 7) von deutlichen Septalrandspinae. Jenes Exemplar (Bayerische Staatssammlung für Paläontologie und Historische Geologie, München) gehört jedoch nach eigener Untersuchung (1980) eindeutig zu Palaeocyathus Foerste, 1888 (mit Synonym Enterolasma Simpson, 1900). Die echte Muenstraia hat offenbar - unerwartet früh bereits im Ludlovium apomorph - glattrandige Septen (lamelläre Mikrostruktur sensu Schindewolf 1942: 27), was aber noch durch Kelchstudien zu beweisen bleibt.

Verbreitung: Unteres Ludlovium bis mittleres/oberes Pragium; Mitteleuropa (Deutschland), NW-Afrika (Marokko), Nordamerika (USA, Kanada).

Arten:

Muenstraia franconica n. sp., Ludlovium, Frankenwald (Deutschland)

Muenstraia thuringica n. sp., mittleres/oberes Pragium, Thüringisches Schiefergebirge (Deutschland) und Tafilalt (Marokko)

Muenstraia squarrosa (Sutherland, 1965), unteres Ludlovium, Oklahoma (USA)

Sutherland stellte seine neue Art nur mit Vorbehalt zu Petraia, weil gelegentlich ein Aulos auftritt und deshalb eventuell nähere Beziehungen zu ..Saucrophyllum Philip, 1962" (= Sutherlandinia Weyer, 1972) bestehen, die auch Fedorowski (1984: 90) diskutierte. Gegen die an einer Lokalität zusammen vorkommende Sutherlandinia arbucklensis (Sutherland, 1965) = Sutherlandinia petaloides (Ball \& Grove, 1940) unterscheiden aber noch andere Merkmale (breiter konisches Polypar mit schwächeren Archaeotheca-Rippen, tiefer Kelch mit wenig Tabulae in Position II), sodass es sich wohl eindeutig um zwei deutlich zu trennende Arten handelt.

Muenstraia n. sp. (new laccophyllid gen. and sp. - Pedder 1975: 286, Abb. 10, 11, 13), oberesLochkovium (Pedavis pesavis-Zone), YukonGebiet (NW-Kanada)

Pedder urteilte, dass sein Unikat zum einen Barrandeophyllum Počta, 1902 (sensu auct.) ähnelt, aber keinen Aulos besitzl, und zum anderen Petraia Münster, 1839 (sensu auct.) nahe steht. aber zahlreiche Tabulae in Position I (sensu Sutherland 1965) ausbildet; gegen beide Genera sollten die prominenten Fossulae unterscheiden, die meines Erachtens aber normale Pseudofossulae darstellen. Das Polypar ist eine typische "Petraia“ im alten Sinne. 
Muenstraia franconica $\mathbf{n}$. sp.

Abb. 1A-C, Taf. 1: $3 \mathrm{a}-\mathrm{b}$

pars 1839 Petraia semistriata [n. sp.] - Münster: 43 (nur 1 Paralectotypus: abgebildet in Weyer 1980: Abb. 1/3, 2/1-2); non Taf. 3, Fig. 2 (semistriata-Lectotypus, bisher ohne Schliffpräparation, = problematisches Taxon, nicht congenerisch mit "Petraia" sensu Schindewolf 1931, wo das Polypar als abnormes Individuum gedeutet wurde); non übrige Paralectotypen.

pars 1870 Petraia tenuicosiata Münst. [mit Synonym Petraia semistriata Münst.] - Kunth: 41.

pars 1885 Petraia semistriata Mstr. - Frech: 96.

pars 1931 Petraia semistriata Münster - Schindewolf: 638, Abb. 5-9; non Abb. 1-4 (neu gezeichnet in Weyer 1980: 31, Abb. 3/1-3. Taf. 1, Figs 1-3Sutherlandinia? sp.); non 648, Taf. 52, Fig. 6 (als semistriata-Holotypus designiert, real aber semistriata-Lectolypus).

pars 1956 Petraia radiata Münster, 1839 - Hill: F259. Abb. 175/6a (Kopie Schindewolf 1931); non Abb. 175/6b (radiata-Lectotypus, = Palaeocya thus).

pars 1965 Petraia radiata Münster, 1839 - Ivanovskiy: 65, Abb. 19a (Kopie Schindewolf 1931); non Abb. 19b (radiata-Lectotypus, = Palaeocyathus).

1980 Petraia sp. - Weyer: 30, Abb. 1/3, 2/1-2, Taf. 2, Figs $1-6$, Taf. 3, Figs $1-6$.

non 1981 Petraia semistriata Münster - Hill: F187, Abb. 112/4a-d (Kopie Schindewolf 1931, = Sutherlandinia? sp.).

non 1995 Petraia semistriata Münster - Lin et al.: 377. Abb. 490a-d (Kopie Schindewolf 1931, = Sutherlandinia? sp.).

Diagnose: Langkonische Muenstraia-Art mit adult 18 Großsepten bei 3-5 mm Polypardurchmesser, von denen 14 sowie die zwei langen antiseptalen Kleinsepten zentral verwachsen sind (ohne Aulos-Trend). Die übrigen Kleinsepten erreichen die halbe Länge der Großsepten. Alle Septen sind fadenförmig dünn (bei entsprechend weiten Interseptallumina) und besitzen lediglich peripher an der Archaeotheca dreieckig verbreiterte Septalsockel.

Holotypus: Polypar BGR Berlin Nr. X9100 (Coll. O. H. Schindewolf 1922) - 6 Querschliffe (Dünnschliffe, Präparation O. H. Schindewolf), Original zu Schindewolf 1931 (Abb. 5-9) und Weyer 1980 (Taf. 2, Figs 1-6, Taf. 3, Figs 1-6).

Locus typicus: Schübelberg bei Elbersrcuth (ehemaliger Steinbruch $1.5 \mathrm{~km}$ im NW des Dorfes) $6 \mathrm{~km} \mathrm{E}$ Wallenfels (Frankenwald).

Stratum typicum: Elbersreuther Orthoceratitenkalk, Ludlovium.

Weiteres Material (vom locus typicus und aus stratum typicum): Paratypus Polypar MB Berlin Nr. K.127. (Coll. G. Münster ca. 1835) 2 Querschliffe (Peels), 1 Reststück (Präparation D. Weyer 1978), Paralectotypus von Petraia semistriata Münster, 1839, Original zu Weyer 1980 (Abb. 1/3, 2/1-2).

Beschreibung: Der schon von Schindewolf (vor 1931) präparierte Holotypus ist ein langkonisches, adult gerades Polypar mit vermutlich weitgehend komplettem Kelch und im Ansatz gerade noch erhaltenem Basistalon, der eine leichte „cornute“ Krümmung bewirkt (Cardinalseptum an konvexer Seite). Die Maße können nur noch geschätzt werden: Länge $15-20 \mathrm{~mm}$. distaler D ca. $6-7 \mathrm{~mm}$.

Die erste Beschreibung des Septalapparats stammt von Schindewolf (1931: 636-644. Abb. 5-9, „Petraia semistriata“); seine Zeichnungen wurden später leicht korrigiert (Weyer 1980: 31, Taf. 2-3). Die insgesamt caliculare Querschliffserie wurde vor der generellen Anwen-

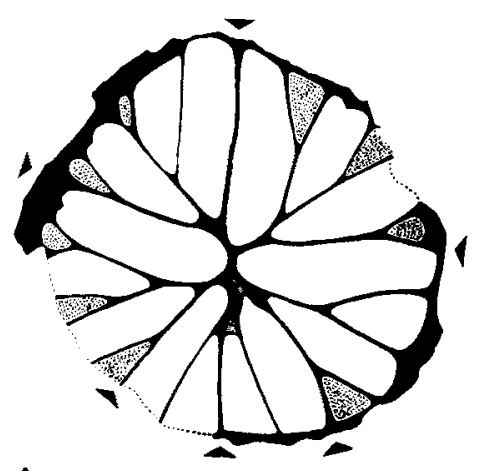

A

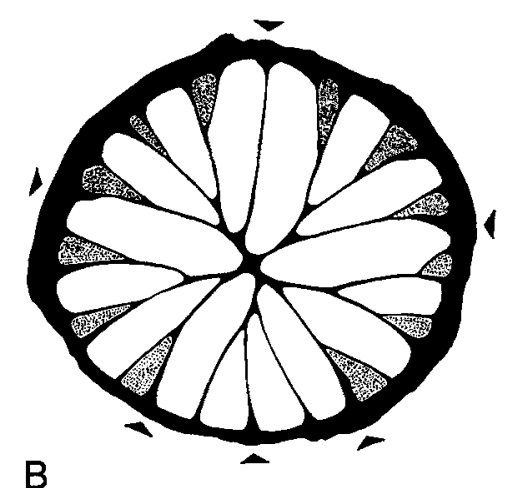

B

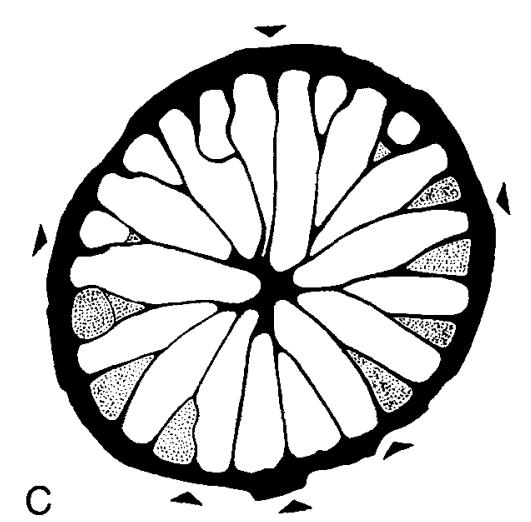

Abb. 1. Muenstraia franconica n. sp., Holotypus (BGR Berlin Nr. X9100); Elbersreuther Orthoceratitenkalk (Ludlovium). Schübelberg bei Elbersreuth (6 km E Wallenfels, Frankenwald). A-C. Caliculare Querschliffe des unteren Kelch (supratabulare Bereiche weiß, subtabulare Anteile punktiert), $\times 14, \times 12$ und $\times 10$ (Fotos Taf. $1,3 a-b$ ),

Fig. 1. Muenstraia franconica n. sp., holotype (BGR Berlin no. X9100); Elbersreuth-Orthoceratites-Limestone (Ludlovian), Schübelberg near Elbersreuth (6 km E of Wallenfels, Upper Franconia). A-C. Calicular cross sections of the lower calice (supratabular areas white, subtabular regions dotted), $\times 14, \times 12$, and $\times 10$ (fotos Pl. 1, 3a-b). 
dung der Peeltechnik angefertigt und erfasst den oberen Kelch nicht. Wegen starker Rekristallisationen ist das Exemplar für Untersuchungen zur Septenmikrosı ruktur ungeeignet.

Der höchste Querschliff (Taf. 1: 3b) zeigt überwiegend kurz: Septen, die längenmäßig gerade in Groß- und Kleinsepten differenziert sind. Nur im linken Artiquadranten hat bereits ein Längenwachstum bis zum Polyparzentrum stattgefunden. Das Cardinalseptum ist im Vergleich zu den benachbarten Großsepten nicht verkürzt. Im tiefer sich anschließenden Stadium (Abb. 1C) liegt bereits der Standardbauplan vor mit axial verwachsenen $\mathrm{G}$ oßsepten, langem Cardinalseptum. großer antiseptaler Triade und regelmäßig ausgebildeten cont atingenten Septenpaaren. Lumina der Position I (sensu Sutherland 1965) sind zum großen Teil bereits subtabular; einmal ist eine zentrifugal geneigte Tabula angeschnitten. Die Triade am Artiseptum bleibt noch länger calicular und verm ttelt so etwas zwischen dem biformen Tabularium-Baustil von Position I und II. Der nächste Querschliff (Abb. 1B. Taf. 1. 3a) repräsentiert diıs typische adulte Muenstraia-Stadium des unt ren Kelchs mit sehr grazil gebauter Axialzone und überall (mit Ausnahme der Antiseptum-Triadt) nicht mehr bewohnten. sondern schon subtabular abgekapselten kleinen Interseptalräumen der markant contratingenten Septenpaare - dies : gehören vorwiegend zur Position I, teilweise an den 4 Insertionsstellen) aber auch zur Position II. Kleinsepten erreichen halbe Großseptenlänge.

In allen Querschliffen bleiben die Septenstellung dominant radial und die axiale Septenverbindung stabil (dünn, nirgends massiv mit stereoplasmalischen Verdickungen). Erste contratingente Fleinsepten setzen bei $\mathrm{D} 2.0$ bis $2.7 \mathrm{~mm}$ ein, sind aber dort noch keineswegs überall angelıgt (Weyer 1980: Taf. 3. Figs 1-2). Alle Septen sind fadenförmig dünn (bei proportional sehr treiten Interseptalräumen) und haben nur direk $t$ an der Wand einen dreieckig verbreiterten Sockel. Tabulae der Position II wurden nicht beobachtet.

Septenformeln:

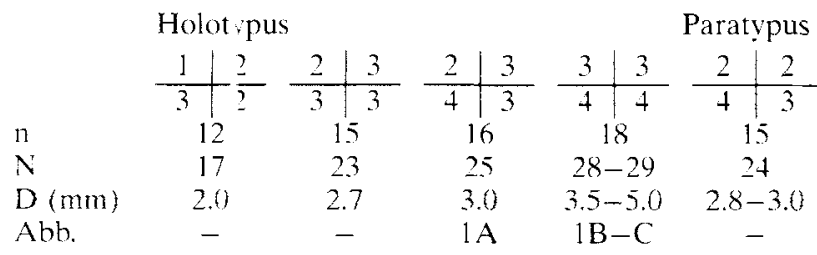

Der Paratypus ist bisher (Weyer 1980) nur geringfügig geschliffen worden, um die wertvollen Petraia-Syntypen der Collection Münster zu schonen. Das langkonische gerade Exemplar (Länge $9 \mathrm{~mm}, \mathrm{D}$ distal $4 \mathrm{~mm}$, proximal $3 \mathrm{~mm}$, dort bereits von Schindewolf angeschliffen) besitzt nach den zwei verfügbaren Querschliffen einen mit dem Holotypus übereinstimmenden Septalapparat, dessen axiale Septenfusion allerdings kleine Lumina aufweist (vermutlich vorübergehender unbeständiger Aulos-Ansatz).

Unterscheidend ist jedoch die wesentlich dünnere Archaeotheca mit deutlichen longitudinalen Septalfurchen, deren Gestalt zudem noch sehr variabel ausfällt (breite Furchen bis schmale Rinnen - Weyer 1980: Abb. 2/2, rechter Antiquadrant). Schindewolf (1931) hatte schon betont, dass dieses noch bei Münster (1839) und Frech (1885) zur Art-Definition verwendete Kriterium an einem Polypar extrem schwanken kann. Die Zuordnung des Paratypus zu Muenstraia franconica ist wegen des Fehlens der dicken Wand möglicherweise nicht ganz sicher; für ein definitives Urteil gibt es leider keine ergänzend zu untersuchenden Topotypen. Weniger kritisch und als intraspezifisch variabel interpretierbar erscheint der abweichende Verlauf der Tabulae in Position I (zentripetal ansteigend beim Holotypus, abfallend beim Paratypus).

Beziehungen: Die Unterschiede zu Muenstraia thuringica $\mathrm{n}$. sp. sind dort genannt. Muenstraia squarrosa (Sutherland, 1965) weicht in der Polypargestalt ab (breitkonisch, nicht langkonisch/subzylindrisch) und hat etwas mehr Septen, sodass die Interseptalräume proportional merklich kleiner sind. Muenstraia sp. (Pedder, 1975) mit ebenfalls höherer Septenanzahl entfernt sich durch ihre längeren Kleinsepten - im Querschliffbild treten lang-dreieckige contratingente Septenpaare auf.

Bemerkungen: Keine der 5 Petraia-Arten von Münster (1839) aus dem Ludlovium von Elbersreuth und aus dem oberen Famennium von Schübelhammer ist im Sinne ihres jeweiligen Art-Typus, die alle eingesehen werden konnten, mit diesem Taxon identisch, sodass eine Neubenennung nötig war.

Petraia radiata Münster, 1839 sensu LudloviumLectotypus (designiert Schindewolf 1931. fälschlich als Neotypus) = Palaeocyathus radiatus (Münster, 1839).

Petraia decussata Münster, 1839 aus dem Oberfamennium (gemäß Lectotypus-Fixierung: Weyer 2000 ) ist die echte Typusart der Gattung. 
Petraia semistriata Münster, 1839 sensu Ludlovium-Lectotypus (designiert Schindewolf 1931, fälschlich als Holotypus) = nach einem unregelmäßigen Querbruchbild im unteren Polypardrittel ein unbekanntes Genus (ohne intensive Serienschliffe, deren Anfertigung nicht genehmigt wird, vorerst nicht deutbar).

Petraia tenuicostata Münster, 1839 sensu Lectotypus (designiert Weyer 1997b) = Ober-Famennium-Vertreter des Tabulata-Genus Actinotheca Frech, 1889.

Für Petraia kochi Münster, 1839 wird zurzeit von mir ein Lectotypus aus dem Ober-Famennium vorbereitet.

Das zweite als "Petraia semistriata" bestimmte Polypar, von dem Schindewolf (1931: Abb. 1-4, BGR Berlin Nr. X9099, neu abgebildet in Weyer 1980: Abb. 3/1-3, Taf. 1, Figs 1-3) eine Querschliffserie vorlegte, unterscheidet sich nur in einem einzigen Merkmal (Antiseptum diploseptal aufgespalten) und ist daher als Sutherlandinia sp. abgetrennt worden. Diese neue Art kann vorläufig nur in offener Nomenklatur geführt werden, bis ergänzendes, als Typus geeignetes Material eine umfassendere morphologische Analyse erlaubt. Am Fundort Schübelberg bei Elbersreuth wurde 1991 (gemeinsam mit Prof. Dr. G. Alberti und Dr. T.-C. Hüsken, Hamburg, sowie Dipl.Geol. K. Bartzsch, Saalfeld) einen vollen Tag lang gesammelt - leider fanden sich lediglich 5 schlecht erhaltene, nahezu unbestimmbare Rugosa-Fragmente.

\section{Muenstraia thuringica n. sp.}

Abb. 2A-K, Abb. 3A-H, Taf. 1: 1a-e, 2a-d

1984 ,Petraia“ n. sp. - Weyer: 11, Foto 2/1.

Diagnose: Langkonische Muenstraia-Art mit adult 18 Großsepten bei 3-6 mm Polypardurchmesser, von denen 14 sowie die zwei langen antiseptalen Kleinsepten zentral direkt (ohne AulosAnsatz) verwachsen sind. Die übrigen Kleinsepten erreichen ein Drittel bis zwei Fünftel der Großseptenlänge. Alle Septen sind von der Peripherie bis zum Zentrum gleichmäßig fadenförmig dünn und umschließen proportional sehr breite Interseptallumina.

Holotypus: Polypar MB Berlin Nr. K.192. (Coll. H. Jaeger 1953) - 14 Querschliffe (2 Peels, 12 Dünnschliffe), Abb. 2A-K, Taf. 1: 1a-e.

Locus typicus: Schadertal (Weganschnitt im NW des Dorfes) $8 \mathrm{~km} \mathrm{~S}$ Saalfeld (Thüringisches Schiefergebirge).
Stratum typicum: Tentakulitenknollenkalk (oberer Teil) $=$ mittleres bis oberes Pragium. Nowakia acuaria-Zone.

Weiteres Material: Paratypus Polypar MB Berlin Nr. K.654. (Coll. D. Weyer 2000) - 12 Querschliffe (8 Peels, 4 Dünnschliffe). Abb. 3A-H, Taf. 1: 2a-d. Nordwestliche Region der Ouidane Chebbi (Profil Azhguig, Mergelkalke) SE Erfoud (Tafilalt, NE-Marokko); nach Geländesituation Lochkovium-Pragium (mit Hercynella), nach Lithologie (graue bis rötliche Kalke) Pragium, nach der zur stratigraphischen Kontrolle aufgesammelten Begleitfauna (Trilobita, Tentaculitida - Determination durch Prof. Dr. G. Alberti, Hamburg, aufbewahrt im MB Berlin) mittleres Pragium.

Die Taxa Reedops cf. cephalotes hamalagdianus G. Alberti, 1983, Crotalocephalina gibba auster G. Alberti, 1970, Odontochile sp., Tropidocoryphe cf. hamalaghdadica G. Alberti, 1967 und Paralejurus cf. hamalaghdadicus G. Alberti, 1983 in der Trilobiten-Assoziation sind nach ihm für das ältere (bis ,mittlere“) Tafilalt-Pragium typisch. Die Tentaculiten-Fauna enthält: Nowakia (Turkestanella) acuaria cf. acuaria (Richter, 1854), Nowakia (Turkestanella) aff. anteacuaria G. Alberti, 1993, Nowakia (Turkestanella?) aff. hercyniana G. Alberti, 1993 [vel Nowakia (Turkestanella) anteacuaria G. Alberti, 1993, später Morphotyp], Peneauia biannulata (Péneau, 1928), Viriatelloides cf. pygmaeus minutulus G. Alberti, 1998 und belegt "mittleres“ Pragium bis (tiefes) Ober-Pragium.

Beschreibung: Der Holotypus ist ein langkonisches, fast subzylindrisches gerades Polypar von $33 \mathrm{~mm}$ Länge mit komplett erhaltenem Kelch (distaler D $10,8 \times 8,5 \mathrm{~mm}$ ), jedoch ohne Spitze (proximaler D 3,4 mm); basal war offenbar ein Anheftungstalon wie beim Paratypus oder wie bei Muenstraia franconica $n$. sp. vorhanden (angedeutet im rechten Cardinalquadranten von Abb.2A). Der Kelch nimmt zwei Drittel der vorliegenden Polyparlänge ein (rekonstruierte Kelchtiefe demnach etwa $50 \%$ ). Die nur lokal gut erhaltene Archaeotheca trägt extern zarte Anwachsrugae und sehr schwache, kaum wahrnehmbare interseptale Längsrippen.

Direkt am Kelchrand (Abb. 2J) ist die dort noch dünne Wand gut septal gegliedert (niedrige, breit gerundete Septenvorsprünge, schmale Interseptalkerben, ohne morphologische Differenzierung in Groß- und Kleinsepten). Tiefer findet allmählich eine Verdickung der Archaeotheca statt, die vorübergehend in fast der gesamten 


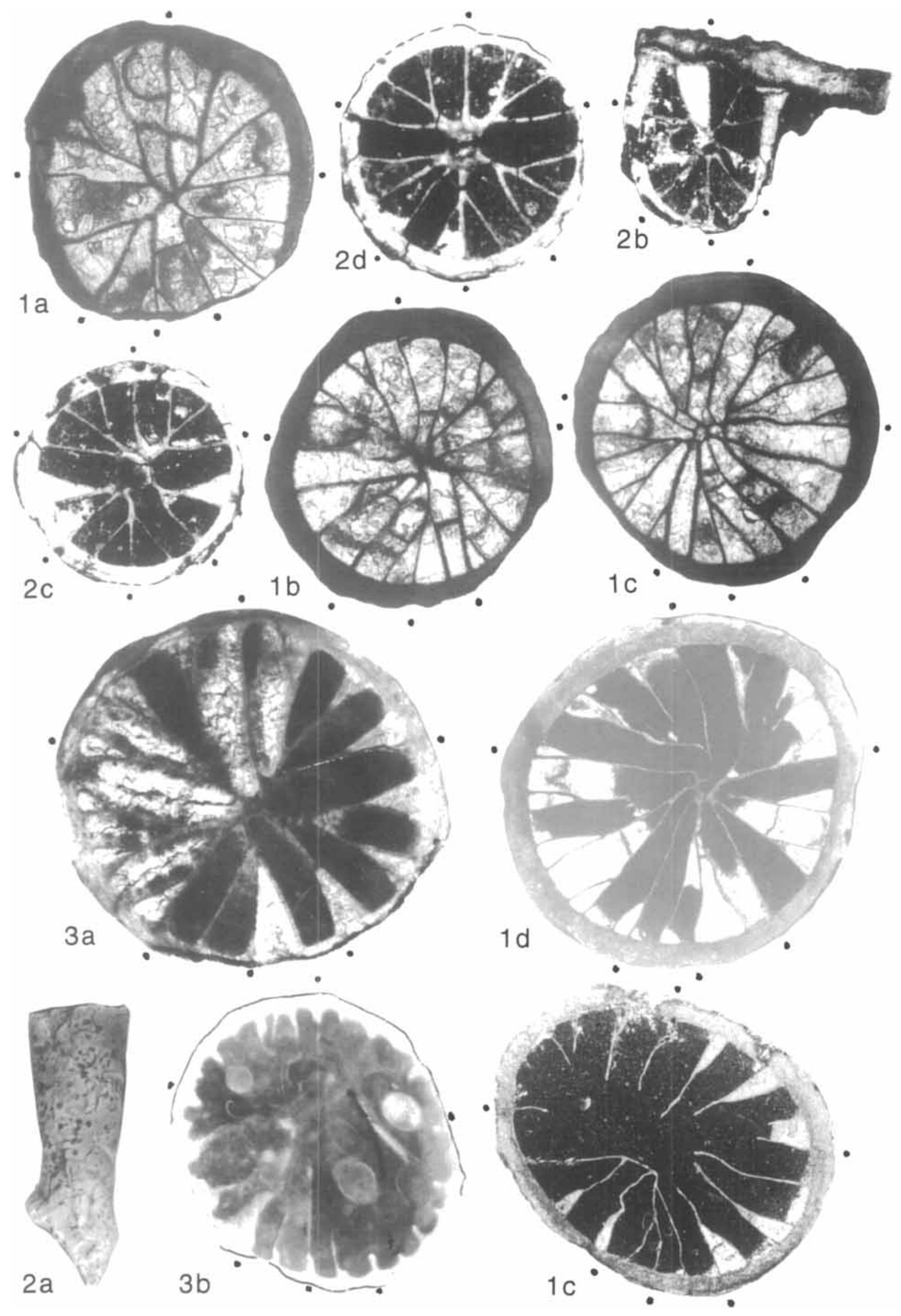




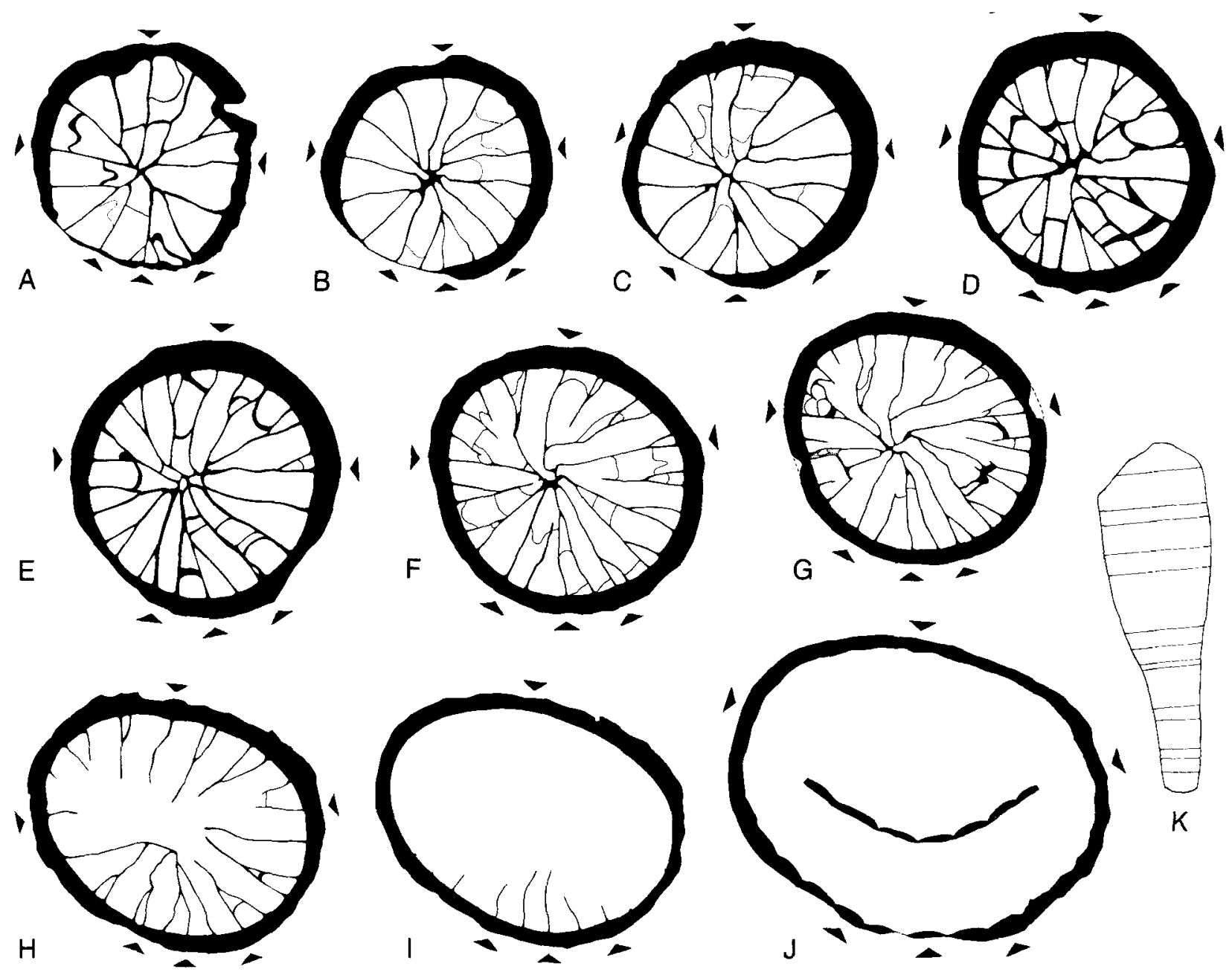

Abb. 2. Muenstraia thuringica n. sp., Holotypus (MB Berlin Nr. K.192.); oberer Tentaculitenknollenkalk (mittleres-oberes Pragium), Schadertal $8 \mathrm{~km} \mathrm{~S}$ Saalfeld (Thüringisches Schiefergebirge). A-J. Querschliffserie (Nr. 1-9, 13, 14) subtabular $(A-E)$, Kelchbasis (F) und calicular (mit kleinen subtabularen Anteilen in Position 1 von $\mathrm{G}, \mathrm{H}): \times 9(\mathrm{~A}-\mathrm{C}), \times 8(\mathrm{D}, \mathrm{E}), \times 7$ (F, G), $\times 6(\mathrm{H}, \mathrm{I})$ und $\times 5(\mathrm{~J})$. Fotos der Schliffe von A, D-F, H - Taf. 1, Fig. 1a-c. In Abb. 2J (Querschliff Nr. 13) ist zentral der unvollständige distalc Qucrschliff Nr. 14 eingefügt. K. Polyparskizze mit eingetragener Position der angefertigten 14 Querschliffe, $\times 1.5$.

Fig. 2. Muenstraia thuringica n. sp., holotype (MB Berlin no. K.192.); upper Tentaculitid-Limestone (middle-late Pragian). Schadertal $8 \mathrm{~km} \mathrm{~S}$ of Saalfeld (Thuringia). A-J. Series of cross sections (no. 1-9.13.14) subtabular (A-E). at base of calice $(\mathrm{F})$, and calicular (with small subtabular areas in position 1 of $\mathrm{G}, \mathrm{H}) ; \times 9(\mathrm{~A}-\mathrm{C}), \times 8(\mathrm{D}, \mathrm{E}), \times 7(\mathrm{~F}, \mathrm{G}), \times 6(\mathrm{H}, \mathrm{I})$, and $\times 5(\mathrm{~J})$. Fotos of sections A. D-F. H - Pl. 1. Fig. la-e. The incomplete distal cross section no. 14 is inserted into the cross section no. 13 (Fig. 2J). K. Sketch of the specimen, indicating the position of the prepared 14 cross sections, $\times 1.5$.

Tafel 1. 1. Muenstraia thuringica n. sp., Holotypus (MB Berlin Nr. K.192.); oberer Tentaculitenknollenkalk (mittleres-oberes Pragium), Schadertal $8 \mathrm{~km} S$ Saalfeld (Thüringisches Schiefergcbirge). a-e. Querschliffe, subtabular und calicular: $\times 15$ (a). $\times 12$ (b-d) und $\times 9$ (e); Zeichnungen derselben Schliffe - Abb. 2A, D-F, H. 2. Muenstraia thuringica n. sp., Paratypus (MB Berlin Nr. K.654.); mittleres Pragium, Ouidane Chebbi (Profil Azhguig) SE Erfoud (Tafilalt). a. Polypar in Seitenansicht. $\times 5.5$. b-d. caliculare Querschliffe, $\times 15$ (Zeichnungen Abb. 3B, G; der nicht gczcichnete Querschliff Fig. $2 \mathrm{c}$ liegt zwischen Abb. 3D-E). 3. Muenstraia franconica n. sp., Holotypus (BGR Berlin Nr. X9100); Elbersreuther Orthoccratitenkalk (Ludlovium). Schübclberg bei Elbersreuth $(6 \mathrm{~km}$ E Wallenfels, Frankenwald). caliculare Querschliffe im unteren (3a, Zeichnung siehe Abb. 1B) und mittleren Kelch (3b), $\times 20$ und $\times 12$.

Plate 1. 1. Muenstraia thuringica n. sp., holotype (MB Berlin no. K.192.); upper Tentaculitid-Limestone (middle-late Pragian), Schadertal $8 \mathrm{~km} \mathrm{~S}$ of Saalfeld (Thuringia). a-e Cross sections, subtabular and calicular; $\times 15$ (a), $\times 12$ (b-d) und $\times 9$ (e): drawings in Fig. 2A, D-F, H. 2. Muenstraia thuringica n. sp., paratype (MB Berlin no. K.654.): middle Pragian. Ouidane Chebbi (section Azhguig) SE of Erfoud (Tafilalt). a. Lateral view of corallum, $\times 5.5$. b-d. Calicular cross sections. $\times 15$ (drawings in Fig. 3B, G; the cross section of Fig. 2C is situated between Fig. 3D-E). 3. Muenstraia franconica n. sp., holotype (BGR Berlin no. X9100); Elbersreuth-Orthoceratites-Limestonc (Ludlovian). Schübelberg near Elbersreuth (6 km E of Wallenfels, Upper Franconia). Calicular cross sections in the lower (3a, drawing Fig. 1B) and in the middle calice $(3 \mathrm{~b}), \times 20$ and $\times 12$. 
oberen Kelchhälfte (Abb. 2I. Cardinalquadranten: nicht atgebildete Querschliffe 10-12 in Abb. $2 \mathrm{~K}) \mathrm{zu}$ einem scheinbar septenfreien Stadium führt. Danach setzen im unteren Kelch erneut Septen ein (als exakte Fortsetzung der Septalvorsprünge am distalen Kelchrand). diesmal in extrem grazilk $r$, von der Wand bis zum Polyparzentrum gleirhmäßig dünner Ausbildung. Die meisten der radial angeordneten Großsepten verbinden sich axial (ohne massive Stereozone): lediglich die z uletzt inserierten aller Quadranten bleiben kürze: (und schen üblicherweise fast immer wie contratingente Kleinsepten aus). Das Cardinalseptum ist stets so lang wie die anderen Großsepten der Hauptquadranten und wird in keinem Kelclistadium bevorzugt verkürzt. Contratingente Kleinsepten erscheinen zuerst (Abb. 2A) anı Antiseptum. wo sie 70-75\% der Großseptenlänge erreichen und eine markante Triade bilder. Die übrigen Kleinsepten setzen später bei $4 . . \mathrm{mm} \mathrm{D}$ ein (Abb. 2D): sie bleiben kürzer (30-4)\% der Großseptenlänge). Ihr Fehlen in $\mathrm{Abb} .2 \mathrm{~A}-\mathrm{C}$ ist ein bekanntes sekundäres Phänomen (..Reduktion“ der primär am jugendlichen Kelchrand inserierten. noch nicht contratingenten Klsinsepten durch Inkorporation in die sich verdickende Wand). Der Septalapparat zeigt folgend. Entwicklung:

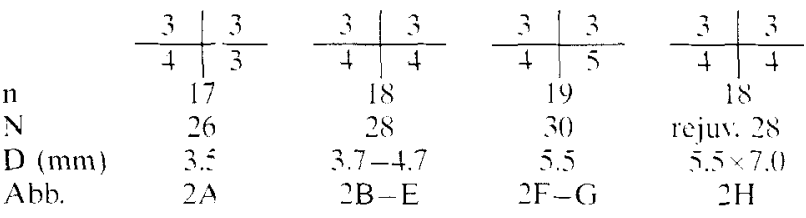

Während det Ontogenese sind pseudofossulare Interseptalräıme nur ausnahmswcise (Abb. 2A) nach Septeninsertionen erkennbar. bevor dic dann vorube gehend teilweise pinnate Großseptenstellung n.ich weiterem Längenwachstum wieder betont radial ausgerichtet wird. Die partielle Auflösung cer axialen Septenverbindung in Abb. $2 \mathrm{~F}-\mathrm{G}$ liängt mit einer hier liegenden Verjüngungsphase zusammen. Die Septenmikrostruktur ist wahrscheinlich lamellär: Stirn und Flanken alle' Septen sind offenbar glatt (ohne Septalrandsp nae $=$ Trabeculae). Distal am obersten Kelchrand fehlt eindeutig die für viele andere Cyathaxoniina (beispielsweise Duncanella. Neaxon) typi sche multitrabekuläre Zone.

Der Bau des markant biformen Tabulariums lässt sich aurh ohne Längsschliff aus der Querschliffserie e mitteln. Maßgebend sind die Füllung der Interseptallumina mit Sparit oder Detritus (speziell an der Kelchbasis: Taf. 1: 1d) und der Verlauf der stets einfachen Bödenschnitte (konkav oder konvex zur Wand). In Position II (sensu Sutherland 1965) liegen die Tabulae überwicgend flach und leicht zur Wand hin abfallend (mehrfach noch mit deutlichem Anstieg direkt an der Archaeotheca: rechts neben dem Cardinalseptum in Abb. 2C, linker Antiquadrant von Taf. 1: 1d). Öfters kommen aber auch axial leicht konkave Böden vor; das ungewöhnliche Nebeneinander beider Strukturformen demonstriert Abb. 2D. Eine echte Cardinalfossula ist nicht ausgebildet. Die flachen Tabulae der Position I ragen höher in den Kelch hinauf (Taf. 1: le) und sind ebenfalls zentripetal oder zentrifugal geneigt.

Der Paratypus ist ein jugendliches, noch komplett caliculares Exemplar mit ähnlich dicker, extern korrodierter Archaeotheca: Länge $10 \mathrm{~mm}$, D proximal ca. $0.5 \mathrm{~mm}$ (mit Talon $2 \times 4 \mathrm{~mm}$ ) und distal $3.5 \mathrm{~mm}$ (nur unterer Kelch mit axialer Septenverbindung erhalten). Die rekonstruierte Länge bis zum Kelchobcrand dürfte ca. $18-20 \mathrm{~mm}$ betragen haben. Das langkonische Polypar ist gerade und nur durch den Anheftungstalon leicht cornut" (Cardinalseptum an konvexer Seite).

Der Septalapparat seines letzten Stadiums (Abb. 3H, D $3.4 \mathrm{~mm}$ ) entspricht Abb. 2C-D (D $3.9-4.5 \mathrm{~mm})$ des Holotypus. In allen Querschliffen fällt wegen häufigerer Insertionen eine deutlicher pinnate. weniger radiale Großseptenstellung mit schwachen Pseudofossulae auf. Die antiseptale Triade ist von Anfang an ausgebildet (ab D $1.8 \mathrm{~mm}$ ). Die übrigen contratingenten Kleinsepten erscheinen erst im letzten Schliff (Abb. 3H) an 3 Stellen. Die Septenformeln lauten:

$\begin{array}{lc}n & 3 \\ N & 20 \\ D(m m) & 1.8-2.2 \\ \text { Abb. } & 3 \mathrm{~A}-\mathrm{B}\end{array}$

\begin{tabular}{c|r}
3 & 3 \\
\hline 3 & 3 \\
16 \\
24 \\
$2.3-2.5$ \\
$3 C-D$
\end{tabular}

\begin{tabular}{c|c}
3 & $\frac{3}{4}$ \\
\hline 4 & 48 \\
28 \\
$2.9-3.4$ \\
$3 E-H$
\end{tabular}

Tabulae wurden nur zweimal basal in Position II beobachtet - sie fallen zur Wand hin ab (Abb. 3A. C). Subtabulare Anteile liegen außerdem in den zwei kleinsten Lumina der Antiquadranten beim zuletzt inserierten Großseptum vor (Abb. 3E). Interseptalräume der Position I (Abb. $3 \mathrm{H}$ ) sind noch calicular. Ein zentrales Lumen (Abb. 3G) könnte eventuell ein ephemerer Aulos-Ansatz sein. Das axiale Lumen in Abb. 3E dagegen ist kein Aulos, sondern ein subtabularer Raum, der durch eine steil stehende, nur im Polyparzentrum vorhandene und im nächsten tieferen Querschliff wieder verschwundene Tabula begrenzt wird. 

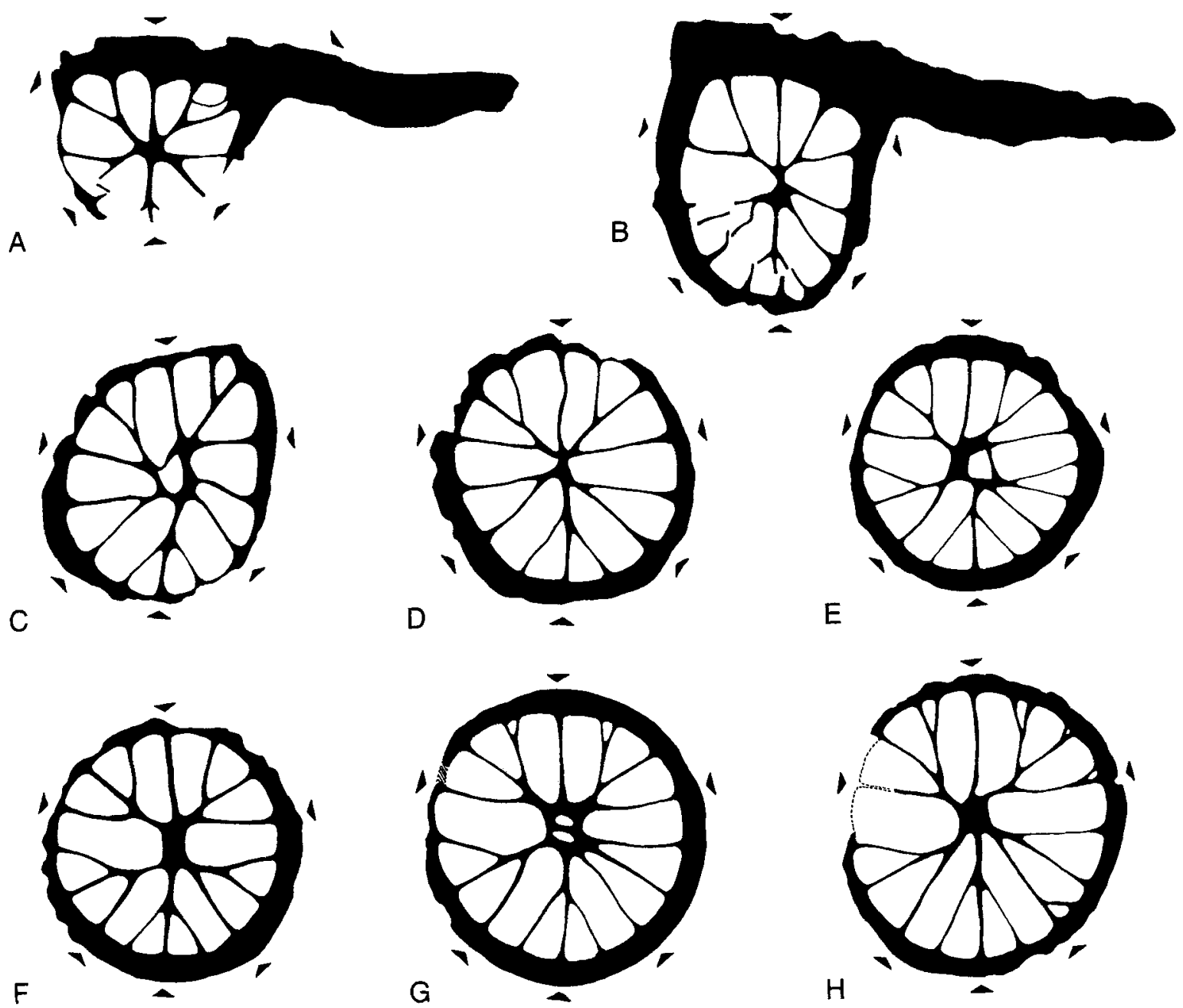

$E$

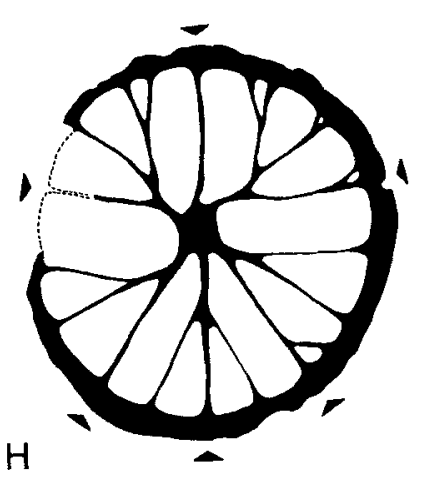

Abb. 3. Muenstraia thuringica n. sp., Paratypus (MB Berlin Nr. K.654.); mittleres Pragium, Ouidane Chebbi (Profil Azhguig) SE Erfoud (Tafilatt). A-H. caliculare Querschliffserie, $\times 15(\mathrm{~A}-\mathrm{D})$ und $\times 12(\mathrm{E}-\mathrm{H})$; Interseptallumina fast immer calicular (mit Detritus erfüllt), auch in Position I, subtabular (mit Sparitfüllung) nur vereinzelt basal (5 Lumina in A, links vom Cardinalseptum in B) sowie vorübergehend axial (C, E, G) und je 1 Lumen zwischen den zwei letzten sichtbaren Großsepten beider Antiquadranten (E). Die Abstände vom basalen Querschliff A betragen $2.3 \mathrm{~mm}$ (B), $2.4 \mathrm{~mm}$ (C), $3.2 \mathrm{~mm}$ (D). $5.1 \mathrm{~mm}$ (E), $5.9 \mathrm{~mm}(\mathrm{~F}), 6.9 \mathrm{~mm}(\mathrm{G})$ und $7.5 \mathrm{~mm}(\mathrm{H})$.

Fig. 3. Muenstraia thuringica n. sp., paratype (MB Berlin no. K.654.); middle Pragian, Ouidane Chebbi (section Azhguig) SE of Erfoud (Tafilalt). A $-\mathbf{H}$. Series of calicular cross sections, $\times 15(\mathrm{~A}-\mathrm{D})$, and $\times 12(\mathrm{E}-\mathrm{H})$; interseptal lumina mostly calicular (filled with detritus, also in position I), rarely subtabular (filled with sparite) at base (5 lumina in A, left side of cardinal septum in B), temporarily in axial parts $(C, E, G)$, and interseptal space between the two last visible major septa of both antiquadrants $(\mathrm{E})$.

Beziehungen: Muenstraia franconica n. sp. hat bei gleicher Polypargestalt peripher nicht so fadenförmig dünne Septen, sondern wandnah leicht verdickte Septensockel. Muenstraia squarrosa (Sutherland, 1965) mit ähnlich dünnen Septen unterscheidet sich durch das breitkonische Polypar, den ersten Trend zur Bildung eines Aulos und die merklich längeren Kleinsepten (bei insgesamt etwas höherer Septenanzahl - $\mathrm{n}$ 22-27 bei 5-7 mm D), Muenstraia n. sp. (Pedder, 1975) besitzt ebenfalls mehr Septen (n 25 bei $7 \mathrm{~mm} \mathrm{D)}$ und deutlich längere Kleinsepten, die oft zwei Drittel der Großseptenlänge erreichen.
Bemerkungen: Die artliche Identität von thüringischem Holotypus und marokkanischem Paratypus erscheint vielleicht etwas gewagt angesichts zweier Unikate. Die für bessere ArtDiagnosen erforderlichen Populationen stehen aber bei diesen seltenen, bisher kaum beschriebenen Formenkreisen nirgends zur Verfügung.

\section{Danksagung}

Mein Dank gilt Dr. Hermann Jaeger (1929-1992), Berlin. für die lange zurückliegende Überlassung eines seltenen Rugosa-Fundes im thüringischen Tentaculiten-Knollenkalk, Dr. Christian Klug, Tübingen, für gemeinsame Fossilaufsammlungen im Tafilalt, und Prof. Dr. Gerhard Alberti, Hamburg. für 
die freundlicherw ise übernommene Bestimmung von Trilobita und Tentaculitida aus der Begleitfauna einer marokkanischen Pragium-Kuralle. Korrigierende Hinweise bein Review-Verfahren $v$ rdanke ich Prof. Dr. Jürgen Kullmann. Tübingen. und Dr. Stefan Schröder. Köln.

\section{Literatur}

Fedorowski. J. 19,44. Subjectivity in the Evaluation of Diag. nostic Characters and its Influence on the Taxonomy of the Rugose Corals. - Palaeontographica Americana 54: $86-91$.

- 1986. Diffingii u. a new suborder of the Rugose corals from SW Teras. - Acta Palacontologica Polonica 30 $(3-4)$ : 209-2-0 (dated 1985).

Frech. F. 1885. Die Korallenfauna des Oburdevons in Deutschiand. - Zeitschrift der Deutschen Geologischen Gesellschaft 3" (1): 21-130.

Hill. D. 1956. Rugosa. Heterocorallia. - In Moore. R. C. (ed.): Treatise on Invertebrate Paleontology. Part F. Coelenterata: I->X. 1-798 (F233-327): Geological Societs of America, New York. University of Kansas Press. Lawrence/Kansas.

- 1981. Rugosa und Tabulata. - In Teichert. C. (ed.): Treatise on Invertebrate Palcontology. Part F. Coelenterata. Supplement 1 1-762 (2 volumes): Geological Society of America. Bol lder/Colorado. University Press. Lawrence/ Kansas.

Ivanovskiy. A. B. 1959. K voprosu o sistematicheskom polozhenii ordovik: kikh i siluriyskikh zafrentoidnykh korallow. [On the syste natical position of Ordovician and Silurian zaphrentoidid corals]. - Doklady Akademii Nauk SSSR 125 (4): $895-399$. [In Russian].

- 1963. Rugozy ordovika i silura Sihirskoy platformy. [Rugosa from th: Ordovician and Silurian of the Siberian platform]. - Institut Geologii i Geofiziki. Akademisa Nauk SSSR. Sibirskoe Otdelenic: 1-160): Moskia (I Ldatel'stvo Akadımii Nauk SSSR). [In Russian].

- 1965. Drevneyshic rugozy. [The oldest Rugosa]. - Institut Geologii i Grofiziki. Akademiva Nauk SSSR. Sibirskoe Otdelenie: 1-1.52: Moskva (Izdatel'stro Nauka). [In Russian!.

Kunth. A. 1870. Beiträge zur Kenntniss fossiler Korallen. 3-5. - Zei schrift der Deutschen Geologischen Gesellschaft 22(1): $24-43$.

Lin Bao-yu. Xu Shou-yong. Jia Hui-zhen. Guo Sheng-zhe. Ouyang Xuan. Wang Zeng-ji. Ding Yun-jie. Cao Xuanduo. Yan Youl-vin \& Chen Hua-cheng 1995. Monograph of Palaeozoi: corals. Rugosa and Heterocorallia. 1-778: Geok gical Publishing House. Beijing. [In Chinese with English : ummary].

Lin Ying-dang \& Liu Peng-ju 1999. The systematic position of the solid column of Rugose coral. - Acta Palaeontologica Sinica 38 (2): 218-227. [In Chinese with English summaryl.

Münster. G. 1830. Der Chiton priscus und einige andere seltene Versteinerungen aus der Vebergangs-Formation. Beiträge zur Petrefacten-Kunde 1: $38-4+$ : Bayreuth (Buchner).

Pedder. A. E. H. 1975. Sequence and relationships of three Lower Devolian coral faunas from Yukon Territory. Paper. Gcolo sical Survey of Canada 75-1 (B): 285-295.
Schindewolf. O. H. 1931. On the genotype and septal development of the coral genus Peiraia Münster. - Quarterly Joumal of the Geological Society of London 87 (4): $6.30-6+9$.

- 1942. Zur Kenntnis der Polycoelien und Plerophyllen. Eine Studie über den Bau der „Tetrakorallen“ und ihro Beziehungen zu den Madrcporarien. - Abhandlungen des Reichsamts für Bodenforschung, Neue Folge 204 $1-324$.

Spasskiy. N. Y. (1977): Devonskie rugozy SSSR (sistematika stratigraficheskoe i geographicheskoe znachenie). [Devonian Rugosa of the USSR (systematics, stratigraphical and geographical importancel. - Ministerstvo Vysshevo Srednego Spetsial nogo Obrazovaniya RSFSR; 1-285; Izdatel'stvo Universiteta, Leningrad. [In Russian].

Sutherland. P. K. 1965. Henryhouse Rugose Corals. - Bulletin. Oklahoma Geological Survey 109: 1-92.

Volz. W. 1896. Dic Systematik der fossilen Korallen. Jahres-Bericht der Schlesischen Gescllschaft für vaterländische Cultur. [für 1895| 73 (II. Abtheilung: Naturwissenschaften. a. Sitzungen der naturwissenschaftlichen Section): $101-107$

Weyer. D. 1972. Rugosa (Antho7oa) mit biformem Tabularium. - Jahrbuch lür Geologie (Zentrales Geologisches Institut Berlin). [für 1968] 4: 439-463.

- 1973. Uher Protozaphrentis Yü, 1957 (Anthozoa Rugosa, Mit1elordoviz). - Paläontologische Abhandlungen, Abteilung A. Paläozoologie 4 (4): 695-706.

- 1974. Zur Kenntnis von Rhegmaphyllum Wedekind, 1927 (Anthozoa. Rugosa: baltoskandisches Silur). - Zcitschrift für Geologische Wissenschaften 2 (2): 157-183.

- 1978. Neue Sutherlandiniinae (Rugosa) aus dem skandina vischen Silur und aus dem thüringischen Devon. - Frejberger Forschungshefte, C 342: 91-116.

- 1980. Das silurisch-devonische Rugosa-Genus Petraia Münster 1839. - Freiberger Forschungshefte, C 357 $25-41$.

- 1984. Korallen im Paläozoikum von Thüringen. - Hallesches Jahrbuch für Geowissenschaften 9: 5-33.

- 1996. Nichlaralla sytovae n.g. n.sp. aus dem Lochkovian von Podolien (Anthozoa, Rugosa: Unterdevon, Ukraine). - Abhandlungen und Berichte für Naturkunde. Museum für Naturkunde Magdeburg [für 1995] 19: 83-103.

- 1997a. Hyposepta and diplosepta in the septal apparatus of Rugosa. In Perejón. A. \& Comas-Rengifo, M. J. (eds.). Procecdings of the VII International Symposium on Fossil Cnidaria. Madrid. 1995. Boletín de la Real Socicdad Española de Historia Natural, Sección Geológica 91 $(1-4): 37-52$.

- 1997b. Actinotheca Frech 1889 (Anthozoa. Tabulata) im eurasiatischen Famenne (Obcrdevon). In Avlar, H., Poll. K. \& Strauch. F. (eds.). Beiträge zur Korallenforschung und Museumsarbcit. Festschrift zum 60. Gcburtstag von Prof. Dr. Klemens Dekentorp. Coral Rescarch Bulletin 5: 247-264.

- 2000. Revision der Gattung Czarnockia Rózkowska 1969 (Anthozoa, Rugosa; Oberdevon). - Abhandlungen und Berichte für Naturkunde. Museum für Naturkunde Magdeburg 21: 75-107 (dated 1999).

Yü Chang-ming 1957. On the occurrence of a new rugose coral from the Middle Ordovician of Sinkiang province, N.W. China. - Acta Palaeontologica Sinica 5 (2): 307-324. [In Chinese with English summary]. 